\title{
Variación en el tamaño de los huevos en garzas (Aves: Ardeidae) que anidan en la ciénaga de Birama, Cuba
}

\author{
Dennis Denis Ávila \\ Dpto. Biología Animal y Humana, Facultad de Biología, Universidad de La Habana, calle 25 entre J e I, Vedado, \\ Cuba;dda@fbio.uh.cu
}

\author{
Recibido 14-I-2014. Corregido 27-VII-2014. Aceptado 28-VIII-2014.
}

\begin{abstract}
Egg size variation in egrets and herons (Aves: Ardeidae) nesting in Birama's swamp, Cuba. Intraclutch egg size variation in birds depends on many ecological factors and on the evolutive history of each species. In wading birds, a trend to smaller eggs with laying order has been described, but comparative reports are scarce. In this study, egg size variation patterns were described for nine Egrets and Heron species nesting in Birama' Swamp, Cuba. The patterns were described using external dimensions of 3142 eggs from 1875 nests of Butorides virescens, Bubulcus ibis, Ardea alba, Nycticorax nycticorax, Nyctanassa violacea and four Egretta species, taken in the field between 1998 and 2006. Results showed that eggs were 4.9-10\% of adult weight and had volume variation coefficients between $6-9 \%$. There were no general and consistent interspecies relationship between clutch size and egg sizes. Average volumes tend to get smaller with laying order, but it is not statistically detectable in Butorides and Bubulcus. Last egg was between $0.2 \%$ and $15 \%$ smaller than the first, showing an inverse relationship with it. Intraclutch asymmetry is light in E. thula and fluctuating around null in Bubulcus. Size only predicted laying or hatching order for the last egg, in nests with more than two eggs, with $72.4 \%$ of confidence. Rev. Biol. Trop. 63 (1): 235-248. Epub 2015 March 01.
\end{abstract}

Key words: egg size, intraclutch variation, wading birds breeding, ardeids, brood reduction.

El significado de la variación de tallas en los huevos de las aves ha sido sometido a una considerable discusión a lo largo de la historia (Slagsvold et al., 1984; Arnoldt, 1999; Jover et al., 1993). El tamaño de los huevos de las aves forma parte importante de las estrategias reproductivas y tiene un papel central dentro de las historias de vida (Lack, 1968). En especial, se relaciona directamente con los patrones de asincronía de puesta/eclosión, y de reducción de nidada, cuyo significado evolutivo para las aves altriciales ha sido fuertemente debatido y para la cual se han propuesto 17 hipótesis alternativas (Stolesson, \& Beisinger, 1995). El creciente grupo de evidencias obtenido en las descripciones de algunos grupos y de especies particulares, parecen mostrar que posiblemente no exista una única explicación para este fenómeno en todas las aves.
Las garzas (Aves: Ardeidae) siguen una estrategia de reducción facultativa de nidada (Mock, \& Parker, 1986; Jover et al. 1993) en la cual el tamaño relativo del último huevo puede ser un componente importante (Parsons, 1970; Nisbet, 1978). Se ha asumido que el orden de puesta refuerza la reducción de la nidada, sin embargo, experimentos de intercambio de huevos, en las gaviotas, han mostraron que aun así las mortalidades fueron superiores en los últimos huevos (Slagsvold et al., 1984). Esto evidencia que no es el orden de eclosión en si mismo sino las características del huevo (posiblemente su reserva de nutrientes) lo que provoca la mortalidad diferencial.

La importancia del tamaño del huevo en la biología reproductiva de las garzas, en general, todavía se desconoce y merece mayor atención. En varios trabajos se han descrito algunos de 
los patrones de variación en algunas especies. Por ejemplo, Telfair (1983) describe para los huevos de garzas ganaderas algunos patrones generales de la variación entre dimensiones o según el orden de puesta. Custer y Frederick (1990) establecen, por primera vez, las diferencias intra-nidadas en 145 nidos de tres especies de garzas, y describen que el orden de puesta de los huevos explica entre el $69-79 \%$ de la variación total en su volumen. Jover et al. (1993) hacen un excelente análisis de la variación intra-nidada del tamaño del huevo en Ardea purpurea. En otros trabajos se brindan las descripciones generales de las dimensiones de los huevos (Dusi, 1966; Dickerman, \& Gavino, 1969; Hammatt, 1981; Telfair, 1983; Frederick, \& Collopy, 1988; Baichich, \& Harrison, 1997), pero aún son escasos los trabajos integradores o comparativos en este grupo de aves.

El significado preciso de los patrones de variación intra-nidada de la masa o la talla de los huevos, a pesar de haber sido sometido a varios análisis exhaustivos (Salgsvold et al., 1984; Viñuela, 1997) aún permanece difuso. El tamaño de los huevos varía mucho más que su composición proteica o lipídica en la mayoría de las especies, aportando hasta el 60\% de la variabilidad del contenido energético (Viñuela, 1997) y además, puede jugar un papel más distintivo en la supervivencia de las crías que la misma composición. El tamaño del huevo se ha visto que aumenta la eficiencia de los pichones al estar correlacionada con el éxito de eclosión, con el tamaño al nacer, con la tasa inicial de crecimiento y supervivencia, entre otras tendencias. Sin embargo, la variación intra-nidada también puede ser resultado de restricciones nutricionales, las que, por otra parte, no afectarían el grado de asimetría entre los huevos de una misma nidada (Viñuela, 1997).

El conocimiento de los aspectos reproductivos de las aves acuáticas coloniales es abundante y profundo, pero con una fuerte asimetría geográfica Norte-Sur. En el área del Caribe, a pesar de su importancia regional para la conservación de este grupo, son notablemente escasos trabajos profundos sobre este tema. En humedales cubanos se han realizado algunos aportes en relación a las características básicas de la reproducción de varias especies como Butorides virescens (Denis et al. 1999), Bubulcus ibis (Denis et al., 2003), Egretta rufescens (Denis, \& Ponce, 2006) y sobre la estructura y composición de algunas colonias (Denis, 2001; Denis et al., 2002; Denis, 2006). También se han realizado varios trabajos acerca de los huevos en estas especies (Denis, \& Ponce, 2007; Denis, \& Rodríguez, 2007).

Sin embargo, el volumen de información aún dista mucho de ser suficiente para considerar medianamente conocida la reproducción de estas aves en la región. En el presente trabajo se parte de la hipótesis de que en un grupo como las garzas, heterogéneo en cuanto a las características de sus estrategias reproductivas, el patrón intra-nidada de tallas de los huevos también debe variar entre especies y entre condiciones ambientales. Por ello, el objetivo de la investigación es la descripción de los patrones de variación de la talla de los huevos en las garzas, para lo cual se comparan sus diferencias intra-nidada en nueve especies nidificantes en varias colonias de la ciénaga de Birama, Cuba, que es el mayor humedal deltaico de la región del Caribe.

\section{MATERIALES Y MÉTODOS}

Los datos fueron tomados en seis colonias multiespecíficas de la ciénaga de Birama (Fig. 1) y una colonia de la provincia de La Habana, entre los años 1998 y 2006. Las especies incluidas en el trabajo fueron Butorides virescens (Aguaitacaimán), Bubulcus ibis (Garza Ganadera), Egretta thula (Garza de Rizos), E. caerulea (Garza Azul), E. tricolor (Garza de Vientre Blanco), E. rufescens (Garza Rojiza), Ardea alba (Garzón), Nyctanassa violacea (Guanabá Real) y Nycticorax nycticorax (Guanabá de la Florida).

Los nidos eran marcados con cintas numeradas y seguidos por varios días para comprobar los cambios en su contenido y detectar nuevas puestas o eclosiones, que permitieran establecer el orden de puesta de los huevos. Los huevos eran numerados con un marcador 


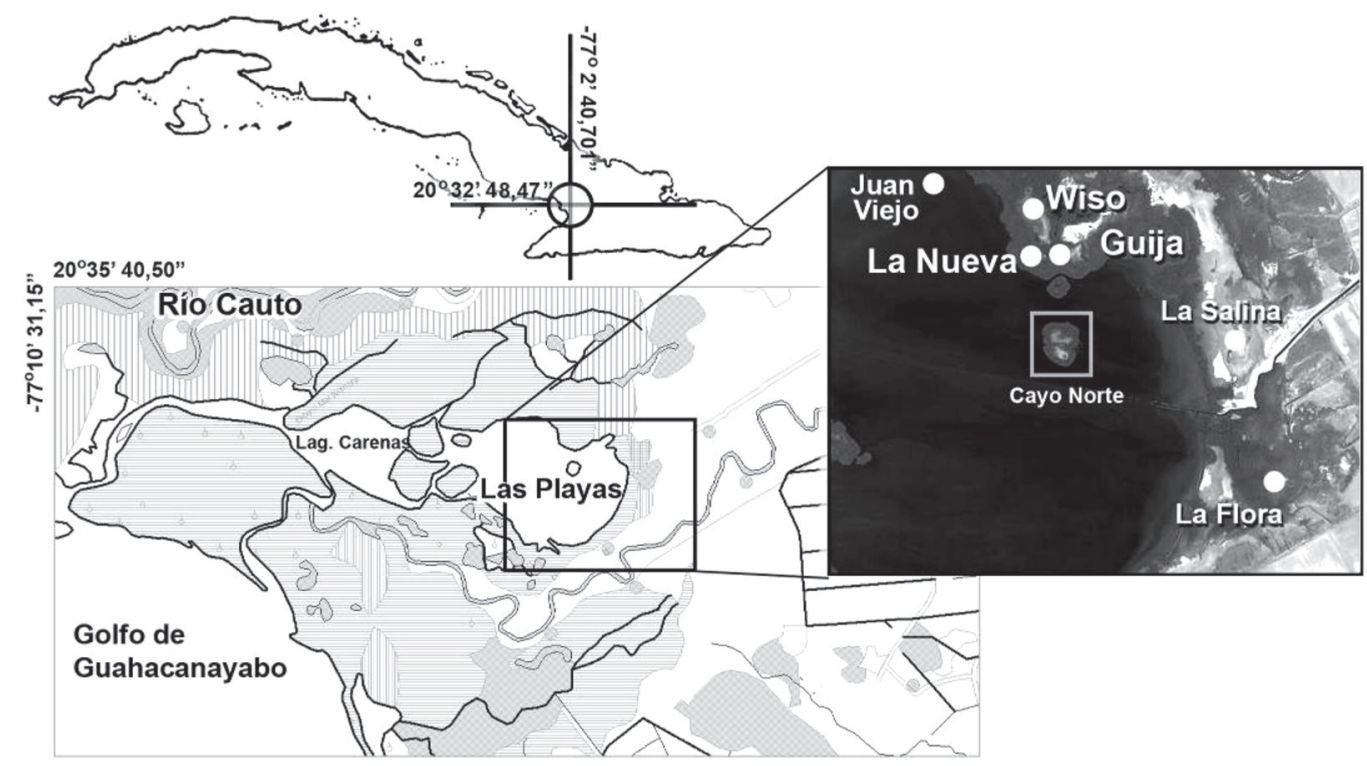

Fig. 1. Ubicación de las colonias de garzas (Aves: Ardeidae) alrededor de la laguna Las Playas, ciénaga de Birama, Cuba, entre 1998 y 2006.

Fig. 1. Location of herons and egrets colonies (Aves: Ardeidae) around Las Playas Lagoon, Birama Swamp, Cuba, between 1998 and 2006.

indeleble no tóxico y se tomaban sus dimensiones externas, diámetro mayor y menor, con un pie de rey de $0.05 \mathrm{~mm}$ de precisión. Con estas medidas se calculó su volumen empleando la fórmula de Hoyt (Hoyt, 1979), pero sustituyendo el coeficiente general dado por este autor, por los coeficientes especie-específicos calculados por Denis et al. (2009) en los casos donde era pertinente. Con la constante general dada por Hoyt $(\mathrm{Kw}=0.548)$ se estimó el peso del huevo fresco. Se calculó, además, un índice de proporción del huevo, definido como la razón diámetro menor/diámetro mayor, para caracterizar aproximadamente la forma del huevo. Como medida de constancia en la forma en cada especie, se empleó el coeficiente de correlación lineal entre las dos variables.

En total, fueron medidos 3142 huevos, pertenecientes a 1875 nidos de las nueve especies (Cuadro 1). Entre el 70 y el $90 \%$ de los nidos en cada especie completaron sus nidadas en el periodo de observación, excepto para Egretta tricolor y Nyctanassa violacea, en las cuales fue solo la mitad. Sin embargo, únicamente en el $20 \%$ de los huevos, su orden de puesta o eclosión, fue observado. El tamaño de puesta real se estableció como el número de huevos de cada nido al final del

\section{CUADRO 1}

Tamaños de muestra empleados para la caracterización morfométrica de los huevos en nueve especies de garzas

(Aves: Ardeidae) en la ciénaga de Birama, Cuba

TABLE 1

Sample sizes of eggs used for morphometric characterization in nine egrets and herons species (Aves: Ardeidae) in the Birama Swamp, Cuba

\begin{tabular}{lcc}
\multicolumn{1}{c}{ Especie } & Nidos & Huevos \\
Butorides virescens & 100 & 234 \\
Bubulcus ibis & 936 & 1156 \\
Egretta thula & 347 & 717 \\
E. caerulea & 36 & 75 \\
E. tricolor & 220 & 422 \\
E. rufescens & 40 & 108 \\
Nyctanassa violacea & 22 & 49 \\
Nycticorax nycticorax & 135 & 303 \\
Ardea alba & 39 & 78 \\
Total & 1875 & 3142 \\
\hline
\end{tabular}


CUADRO 2

Peso corporal medio de adultos empleados para la relativización de las tallas de los huevos en las nueve especies de garzas (Aves: Ardeidae) estudiadas en la ciénaga de Birama, Cuba

TABLE 2

Adult mean body weight used to relativize egg sizes in the nine species of egrets and herons (Aves: Ardeidae) studied in the Birama Swamp, Cuba

\begin{tabular}{lcccc}
\multicolumn{1}{c}{ Nombre científico } & Siglas & Peso corporal & (n) & Fuente \\
Butorides virescens & GRHE & 181.2 & 23 & Acosta et al. (2001) \\
Bubulcus ibis & CAEG & 323.4 & 45 & Acosta et al. (2001) \\
Egretta thula & SNEG & 354.8 & 45 & Acosta et al. (2001) \\
Egretta caerulea & LBHE & 362.8 & 9 & Acosta et al. (2001) \\
Egretta tricolor & LOHE & 378.5 & 17 & Acosta et al. (2001) \\
Egretta rufescens & REEG & 613.5 & 7 & FMNH, Birds of NA \\
Nyctanassa violacea & YCNH & 649 & 7 & Hartman (1955) \\
Nycticorax nycticorax & BCNH & 780.6 & 13 & Acosta et al. (2001) \\
Ardea alba & GREG & 934.4 & 18 & Acosta et al. (2001) \\
\hline
\end{tabular}

periodo de observación, mientras no existieran pérdidas intermedias.

Con propósitos comparativos se procesaron además las mediciones de 173 huevos de Butorides virescens, A. alba, E. tricolor y $N$. violacea, colectados entre 1939-1960 y depositados en las colecciones del Instituto de Ecología y Sistemática, del Ministerio de Ciencia Tecnología y Medio Ambiente de Cuba.

Se comprobó la normalidad por la prueba de Kolmogorov-Smirnov, y en los casos en que fue necesario, se eliminó el 5\% de los valores extremos (para evitar el efecto de los outliers). Los patrones de talla analizados en los huevos fueron las diferencias interespecíficas, entre años, entre localidades, entre tamaños de puesta y la variación intra-nidada asociada al orden de puesta. Para las comparaciones interespecíficas se relativizaron las dimensiones en función del peso corporal adulto de cada especie (Cuadro 2), y se empleó una prueba de Kruskal-Wallis, seguida de una prueba de comparaciones múltiples de Tukey. La misma prueba fue utilizada en las comparaciones de las dimensiones según el orden de puesta. Como existe un efecto significativo de la hembra sobre las dimensiones de los huevos, estos no son observaciones independientes dentro de un mismo nido (Viñuela, 1997) por lo que en las descripciones por especie se trabajó con las medias por nido y en el análisis del patrón intra-nidada se relativizó al valor promedio de la propia nidada.

En todas las pruebas de hipótesis se asumió un nivel de significación de 0.05 , y como estadístico de dispersión se utilizó el error estándar. El procesamiento estadístico se realizó con el programa Statistica v 8.0 (Statsoft 2007).

\section{RESULTADOS}

Las dimensiones absolutas de los huevos de las ocho especies de garzas estudiadas (Cuadro 3) se relacionaron directamente con el tamaño de las especies adultas (Fig. 2), por lo cual se pueden observar los mismos patrones de similitud interespecífica que en esta otra variable. Cada huevo representó tan solo entre $4.9-8 \%$ del peso adulto. En B. virescens, que es la especie de menor tamaño, los huevos representaron el $5 \%$ del peso corporal, mientras que en E. alba y en N. nycticorax, que son las mayores especies, los huevos solo representaron entre 4.9 y $5.3 \%$ respectivamente. Entre las garzas medianas, los huevos representaron entre el 6 y el $8 \%$, siendo $B$. ibis la que invertía mayor biomasa en el huevo promedio.

En cuanto a las dimensiones lineales, los tamaños extremos correspondieron también a 
CUADRO 3

Dimensiones externas de los huevos de nueve especies de garzas (Aves: Ardeidae) en la ciénaga de Birama, Cuba (media \pm E.S. (min-max)) (ns: no significativa)

TABLE 3

External dimensions of eggs in nine herons and egrets species (Aves: Ardeidae) in the Birama Swamp, Cuba (mean \pm standard error (min-max)) (ns: non significant)

\begin{tabular}{lccccc}
\multicolumn{1}{c}{ Especie } & $\mathrm{N}$ & Diámetro Mayor $(\mathrm{mm})$ & Diámetro Menor $(\mathrm{mm})$ & Volumen $\left(\mathrm{cm}^{3}\right)$ & Correlación e/ diámetros $\left(\mathrm{r}_{\mathrm{s}}\right)$ \\
B. virescens & 234 & $37.34 \pm 0.11(34.00-41.20)$ & $28.67 \pm 0.06(26.30-30.90)$ & $15.64 \pm 0.08(12.08-19.05)$ & 0.207 \\
E. thula & 718 & $42.73 \pm 0.06(39.00-46.70)$ & $31.45 \pm 0.04(28.90-34.20)$ & $21.54 \pm 0.07(16.79-26.73)$ & 0.286 \\
E. caerulea & 75 & $44.00 \pm 0.17(41.00-46.70)$ & $32.61 \pm 0.10(31.10-35.10)$ & $23.83 \pm 0.18(20.54-27.79)$ & $0.037(\mathrm{~ns})$ \\
E. tricolor & 422 & $44.38 \pm 0.08(40.90-48.00)$ & $32.19 \pm 0.05(29.60-35.00)$ & $23.43 \pm 0.08(18.69-28.22)$ & 0.169 \\
B. ibis & 1156 & $45.43 \pm 0.06(40.40-50.40)$ & $32.41 \pm 0.03(29.60-35.80)$ & $24.33 \pm 0.06(18.55-31.40)$ & 0.249 \\
E. rufescens & 108 & $49.27 \pm 0.14(46.60-52.20)$ & $36.01 \pm 0.14(31.80-38.40)$ & $32.57 \pm 0.27(24.86-39.10)$ & $0.121(\mathrm{~ns})$ \\
N. violacea & 49 & $49.27 \pm 0.26(45.70-53.30)$ & $36.95 \pm 0.16(34.90-39.00)$ & $34.30 \pm 0.40(28.33-40.17)$ & 0.330 \\
N. nycticorax & 303 & $51.33 \pm 0.12(46.60-55.70)$ & $36.98 \pm 0.09(33.30-40.30)$ & $35.79 \pm 0.19(28.41-43.72)$ & $0.089(\mathrm{~ns})$ \\
E. alba & 78 & $55.49 \pm 0.24(51.80-61.10)$ & $40.24 \pm 0.13(38.20-42.40)$ & $45.79 \pm 0.38(39.37-52.83)$ & 0.278
\end{tabular}

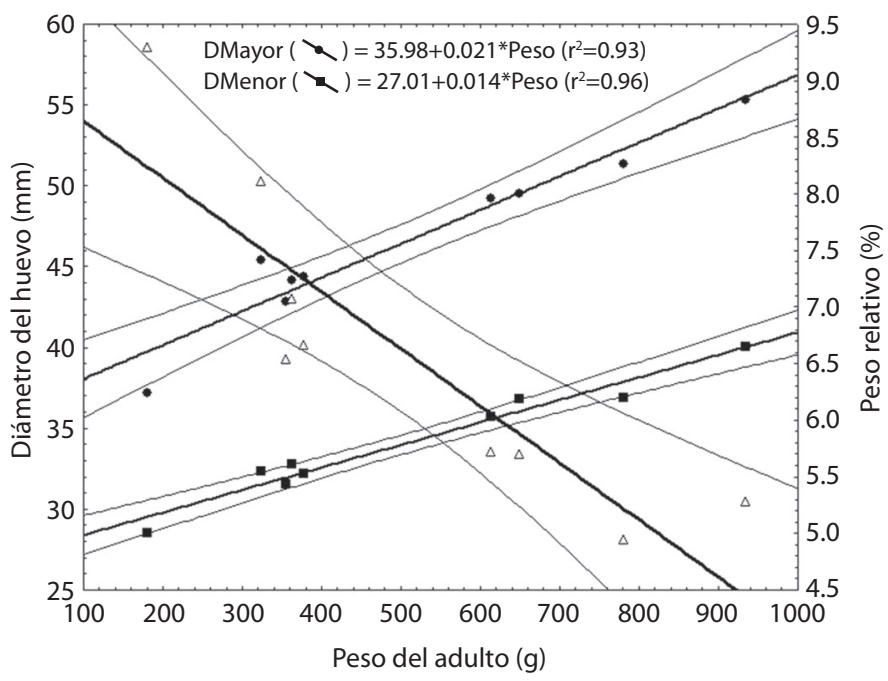

Fig. 2. Relación entre las dimensiones absolutas y el peso relativo del huevo, con el peso corporal de los adultos en ocho especies de garzas (familia Ardeidae) en la ciénaga de Birama, Cuba.

Fig. 2. Relationship between dimensions and relative weight of the egg, with the adult body weight in nine species of egrets and herons (Ardeidae) in Birama Swamp, Cuba.

B. virescens y E. alba, y las garzas de mediano tamaño, B. ibis, E. thula, E. caerulea y E. tricolor, presentaron huevos similares, así como las de tamaño mediano a grande (E. rufescens, Nycticorax y Nyctanassa).

El volumen fue la medida de mayor variabilidad, de entre el 6-9\%. La especie de menor variabilidad en esta medida fue $E$. caerulea
$(\mathrm{CV}=6.2 \% ; \mathrm{n}=30)$ y la de mayor fue $N$. nyctico$\operatorname{rax}(\mathrm{CV}=8.9 \%, \mathrm{n}=115)$. Las dimensiones lineales variaron entre un 3-4\%, siendo el diámetro mayor más variable en todas las especies. Al representar las diferencias en las variabilidades entre los huevos individuales y tomando los promedios por nidada, con lo cual se elimina el efecto de la variabilidad intra-nidada, se 
observaron notables diferencias en diámetro mayor y volumen de los huevos del B. virescens, y en el diámetro menor de los de $E$. rufescens (Fig. 3).

No existió una relación clara y general entre el tamaño de puesta y la talla de los huevos por nido (Fig. 4). En el diámetro menor $N$. nycticorax mostró una clara y marcada tendencia a una disminución en las puestas mayores, mientras que en el diámetro mayor, la alta variabilidad enmascaró cualquier tendencia. Sin embargo, el volumen del huevo también reflejó la tendencia a disminuir en las puestas mayores. En las nidadas de uno a tres huevos, en E. thula, los huevos tuvieron aproximadamente el mismo volumen, aunque

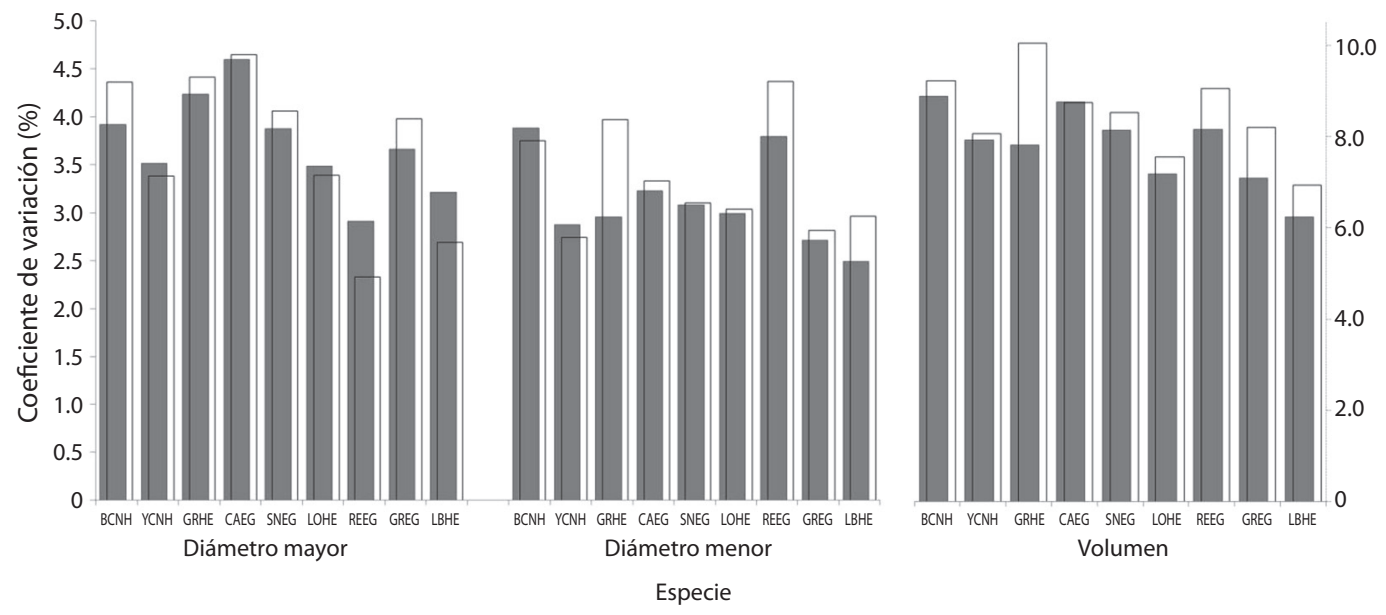

Fig. 3. Variabilidades de las dimensiones de los huevos tomando como unidad de cálculo a los huevos independientes (barras grises, eje Y primario) y a los promedios por nido (barras transparentes, eje Y secundario), en nueve especies de garzas (Aves: Ardeidae) en la ciénaga de Birama, Cuba (las siglas de las especies aparecen en el cuadro 2).

Fig. 3. Variability of the egg dimensions using as unit the independents eggs (gray bars, primary Y axes) and nest average (transparent bars, secondary Y axes), in nine species of herons and egrets (Aves: Ardeidae) in the Birama Swamp, Cuba (species symbols in Table 2).

Tamaño de puesta

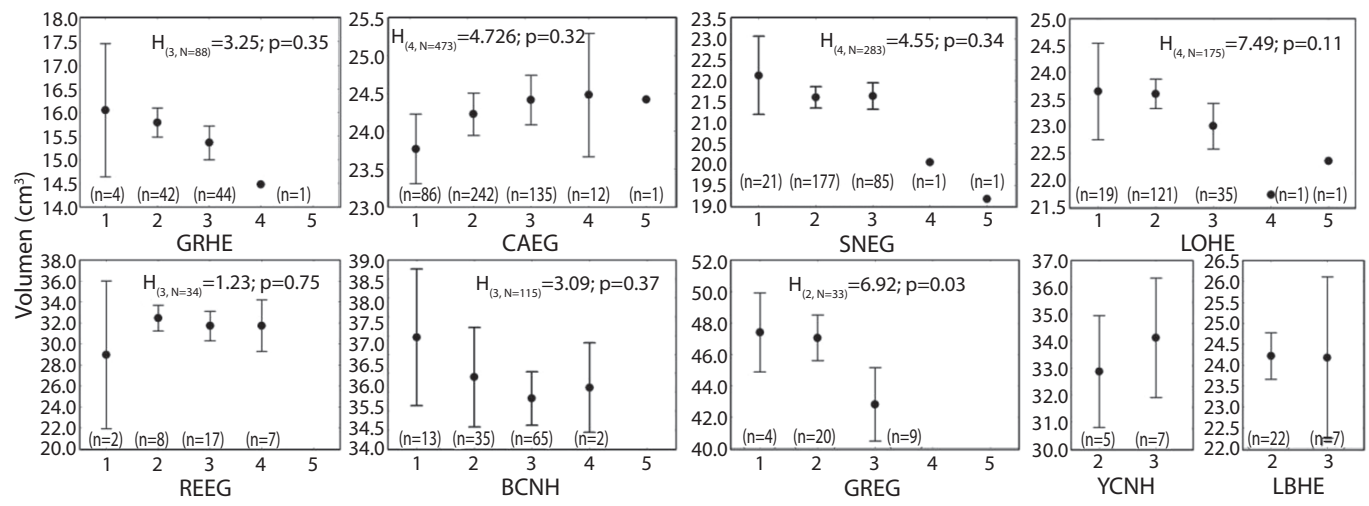

Fig. 4. Volumen promedio de los huevos por nidada según el tamaño de puesta, en nueve especies de garzas (Aves: Ardeidae) en la ciénaga de Birama, Cuba (las siglas de las especies aparecen en el cuadro 2).

Fig. 4. Average volume of eggs per nest according to clutch size, in nine species of herons and egrets (Aves: Ardeidae) in the Birama Swamp, Cuba (species symbols in Table 2). 
en un nido de cuatro y otro de cinco huevos, el tamaño medio fue menor. Este mismo patrón apareció también en E. tricolor. En B. ibis, por el contrario, apareció un patrón diferente, con tendencia al aumento del tamaño del huevo en nidadas mayores, fundamentalmente por un aumento paulatino en los diámetros menores $\left(\mathrm{H}_{(4, \mathrm{~N}=474)}=13.54, \mathrm{p}=0.009\right)$, que compensó la aparente tendencia a la disminución del diámetro mayor $\left(\mathrm{H}_{(4, \mathrm{~N}=476)}=3.144\right.$; $\mathrm{p}=0.53$ ). En $B$. virescens el diámetro menor permaneció similar entre puestas de diferentes tamaños $\left(\mathrm{H}_{(3, \mathrm{~N}=88)}=0.691, \mathrm{p}=0.80\right)$, pero el diámetro mayor disminuyó significativamente $\left(\mathrm{H}_{(3, \mathrm{~N}=88)}=0.691, \mathrm{p}=0.88\right)$.

No en todas las especies de garzas apareció en los huevos un patrón intra-nidada consistente de forma evidente con la estrategia de reducción de nidada (Cuadro 4). El efecto de la alta variabilidad entre hembras (nidos) generalmente oscurece los patrones intra-nidada de los huevos, resultando detectables estadísticamente

CUADRO 4

Dimensiones externas de los huevos, según su orden de puesta, en ocho especies de garzas (Aves: Ardeidae) en la ciénaga de Birama, Cuba (media \pm E.S.)

TABLE 4

External dimensions of eggs, according the laying order, in eight species of herons and egrets (Aves: Ardeidae) in the Birama Swamp, Cuba (mean \pm standard error)

\begin{tabular}{|c|c|c|c|c|c|}
\hline Especie & Orden & $\mathrm{n}$ & Diámetro mayor (mm) & Diámetro menor (mm) & Volumen $\left(\mathrm{cm}^{3}\right)$ \\
\hline \multirow[t]{3}{*}{ B. virescens } & 1 & 17 & $37.7 \pm 0.5$ & $28.6 \pm 0.32$ & $15.6 \pm 0.5$ \\
\hline & 2 & 17 & $36.0 \pm 0.8$ & $28.1 \pm 0.7$ & $14.3 \pm 0.6$ \\
\hline & 3 & 14 & $37.1 \pm 0.3$ & $28.6 \pm 0.4$ & $15.2 \pm 0.4$ \\
\hline \multirow[t]{3}{*}{ E. thula } & 1 & 48 & $43.1 \pm 0.3$ & $31.4 \pm 0.1$ & $21.3 \pm 0.2$ \\
\hline & 2 & 54 & $42.7 \pm 0.3$ & $31.5 \pm 0.1$ & $21.3 \pm 0.3$ \\
\hline & 3 & 30 & $42.4 \pm 0.3$ & $31.8 \pm 0.7$ & $21.9 \pm 1.4$ \\
\hline \multirow[t]{2}{*}{ E. caerulea } & 1 & 4 & $44.9 \pm 0.8$ & $32.6 \pm 0.5$ & $24.0 \pm 0.9$ \\
\hline & 2 & 3 & $44.3 \pm 1.0$ & $33.0 \pm 0.0$ & $24.1 \pm 0.6$ \\
\hline \multirow[t]{4}{*}{ E. tricolor } & 1 & 36 & $44.7 \pm 0.3$ & $32.3 \pm 0.2$ & $23.4 \pm 0.3$ \\
\hline & 2 & 36 & $44.5 \pm 0.3$ & $31.8 \pm 0.2$ & $22.5 \pm 0.4$ \\
\hline & 3 & 13 & $44.2 \pm 0.5$ & $31.4 \pm 0.2$ & $21.8 \pm 0.3$ \\
\hline & 4 & 1 & 41.4 & 29.7 & 18.4 \\
\hline \multirow[t]{4}{*}{ B. ibis } & 1 & 116 & $45.6 \pm 0.3$ & $31.9 \pm 0.2$ & $23.5 \pm 0.3$ \\
\hline & 2 & 91 & $45.4 \pm 0.2$ & $32.3 \pm 0.1$ & $23.8 \pm 0.3$ \\
\hline & 3 & 55 & $44.7 \pm 0.3$ & $31.9 \pm 0.2$ & $22.9 \pm 0.3$ \\
\hline & 4 & 6 & $45.5 \pm 1.0$ & $32.5 \pm 0.8$ & $24.3 \pm 1.5$ \\
\hline \multirow[t]{4}{*}{ E. rufescens } & 1 & 10 & $49.9 \pm 0.5$ & $36.8 \pm 0.3$ & $33.8 \pm 0.7$ \\
\hline & 2 & 14 & $48.9 \pm 0.4$ & $35.7 \pm 0.3$ & $31.3 \pm 0.6$ \\
\hline & 3 & 5 & $49.4 \pm 0.7$ & $33.6 \pm 2.7$ & $28.9 \pm 4.1$ \\
\hline & 4 & 1 & 49.7 & 36.9 & 33.9 \\
\hline \multirow[t]{3}{*}{ N. violacea } & 1 & 3 & $49.0 \pm 0.3$ & $36.4 \pm 0.4$ & $32.6 \pm 0.9$ \\
\hline & 2 & 10 & $50.4 \pm 0.9$ & $37.4 \pm 0.4$ & $35.3 \pm 1.1$ \\
\hline & 3 & 8 & $49.9 \pm 0.6$ & $36.4 \pm 0.8$ & $33.2 \pm 1.2$ \\
\hline \multirow[t]{3}{*}{ N. nycticorax } & 1 & 9 & $52.7 \pm 0.5$ & $37.4 \pm 0.7$ & $37.1 \pm 1.3$ \\
\hline & 2 & 9 & $51.4 \pm 0.6$ & $37.7 \pm 0.4$ & $36.6 \pm 0.8$ \\
\hline & 3 & 20 & $50.5 \pm 0.5$ & $36.7 \pm 0.3$ & $34.1 \pm 0.7$ \\
\hline \multirow[t]{3}{*}{ E. alba } & 1 & 6 & $55.2 \pm 0.4$ & $41.0 \pm 0.4$ & $46.5 \pm 1.1$ \\
\hline & 2 & 8 & $54.7 \pm 0.5$ & $40.4 \pm 0.5$ & $44.8 \pm 1.1$ \\
\hline & 3 & 2 & $52.9 \pm 0.1$ & $39.7 \pm 0.7$ & $41.8 \pm 1.5$ \\
\hline
\end{tabular}


las diferencias solo en el caso del N. nycticorax (Diámetro mayor: $\mathrm{H}_{(2,38)}=8.31 ; \mathrm{p}=0.02$; Volumen: $\left.\mathrm{H}_{(2,38)}=8.64 ; \mathrm{p}=0.01\right)$ y de E. tricolor (Diámetro menor: $\mathrm{H}_{(3,86)}=12.18 ; \mathrm{p}<0.007$; Volumen: $\mathrm{H}_{(3,86)}=11.19$; $\left.\mathrm{p}=0.01\right)$.

Para aumentar la resolución en el análisis del patrón intra-nidada, eliminando el efecto de la variabilidad entre nidos, se compararon los patrones en las dimensiones relativizadas al valor promedio de cada nidada (Fig. 5). Con esta transformación, el patrón intra-nidada de la disminución con el orden de puesta se hace más evidente. Las variaciones en el diámetro menor y mayor se compensan y resultan en volúmenes decrecientes con el orden de puesta. Las mayores diferencias se observaron entre los dos primeros huevos y el tercero.

El tercer huevo en todas las especies fue menor que el promedio de la nidada aunque la magnitud de la diferencia absoluta varió entre especies $\left(\mathrm{H}_{(6, \mathrm{~N}=42)}=14.16 ; \mathrm{p}=0.03\right)$ (Fig. 6A). Al expresar en porcentaje la diferencia entre el primer y tercer huevo para eliminar el efecto del tamaño de los mismos y hacerla comparable entre especies, se observó que, excepto en $B$. ibis, el último huevo es, como promedio, un $8 \%$ menor que el primero (Fig. 6B). En la mayoría de los nidos de B. ibis el tercer huevo fue entre 0.2 y $15 \%$ menor que el primero (promedio: 6.6\%), pero en seis casos fue entre un $3 \%$ y un $15 \%$ mayor, por lo cual no se alcanza significación estadística en general $(\mathrm{t}=0.68 ; \mathrm{p}=0.50 ; \mathrm{gl}=29)$. Del resto de las especies la mayor diferencia intra-nidada apareció en E. tricolor, en la cual, en tres nidos el primer huevo fue $10.8 \%$ mayor que el tercero. La menor diferencia se encontró en E. thula, en la cual el primer huevo solo tuvo un $4 \%$ del volumen mayor que el tercero $\left(Z_{\text {ajust. }}=2.66 ; p=0.008 ; N_{\text {total }}=22\right)$. En las demás especies las diferencias estuvieron alrededor del $8-9 \%$, todas con significación estadística (B. virescens: $\mathrm{Z}_{\text {ajust. }}=1.98 ; \mathrm{p}=0.05 ; \mathrm{N}_{\text {total }}=14$; N. nycticorax: $\mathrm{Z}_{\text {ajust. }}=2.31 ; \mathrm{p}=0.02 ; \mathrm{N}_{\text {total }}=8 ; \mathrm{y}$ E. rufescens: $\mathrm{Z}_{\text {ajust. }}=1.96 ; \mathrm{p}=0.05 ; \mathrm{N}_{\text {total }}=6$ ). En general, existió una relación inversa entre el volumen relativo del primer huevo y el del tercer huevo (Fig. 7) sin embargo, este resultado debe observarse con cuidado ya que dentro de una nidada, los huevos no son observaciones independientes y pueden existir dependencias filogenéticas entre las especies más cercanas, lo cual puede alterar la significación estadística.

En E. alba y $N$. violacea solo se observó un nido con tres huevos en el que se tuvo el orden de puesta y las mediciones de todos. En el primer caso el último huevo fue un $8.76 \%$ menor, y en la segunda especie fue $5.4 \%$ mayor que le primero.

Al comparar las dimensiones de los huevos de cuatro especies entre los datos de la ciénaga de Birama con los de los huevos colectados en diferentes localidades del país, en la primera mitad del siglo pasado y almacenados en colecciones científicas (Fig. 8), no se detectan diferencias significativas en E. tricolor (Diámetro mayor $\mathrm{t}=-1.004 ; \mathrm{p}=0.32$; Diámetro menor $\mathrm{t}=0.22 ; \mathrm{p}=0.82 ;$ Volumen $\mathrm{t}=0.40 ; \mathrm{p}=0.69$; $\mathrm{n}_{1}=48$ y $\mathrm{n}_{2}=399$ ) ni en el diámetro menor de los huevos del E. alba $\left(\mathrm{t}=1.16 ; \mathrm{p}=0.25 ; \mathrm{n}_{1}=11\right.$ y $\left.\mathrm{n}_{2}=74\right)$. En las demás especies se detectaron diferencias significativas que deben reflejar variaciones geográficas o temporales.

El orden de puesta observado y el orden predicho por la talla del huevo, considerando todas las especies, tuvieron una asociación significativa $\left(\mathrm{X}^{2}=84.6, \mathrm{p}<0.001\right)$. En el grupo, en general, la talla puede predecir el orden sólo con un $58.9 \%$ y $52.6 \%$ de confianza para el primer y segundo huevo (prácticamente al azar) y con un $72.4 \%$ para el tercero.

\section{DISCUSIÓN}

En general, el tamaño de los huevos fue similar a lo reportado en otras localidades para cada especie. Las dimensiones de los huevos de $B$. virescens son muy similares a las mencionadas para San Blas, México, (37.5 x 29.2mm; n=232) (Dickerman, \& Gavino, 1969), para New York (38.8 x 29.6mm; $n=74$ ) (Meyerriecks, 1962), y para California (39.0 x 29.5mm; n=17) (Huey, 1926). En N. violacea las dimensiones de los huevos son casi idénticas a las reportadas por Bent (1926) y Palmer (1962) para Norteamérica. Telfair (1983) 

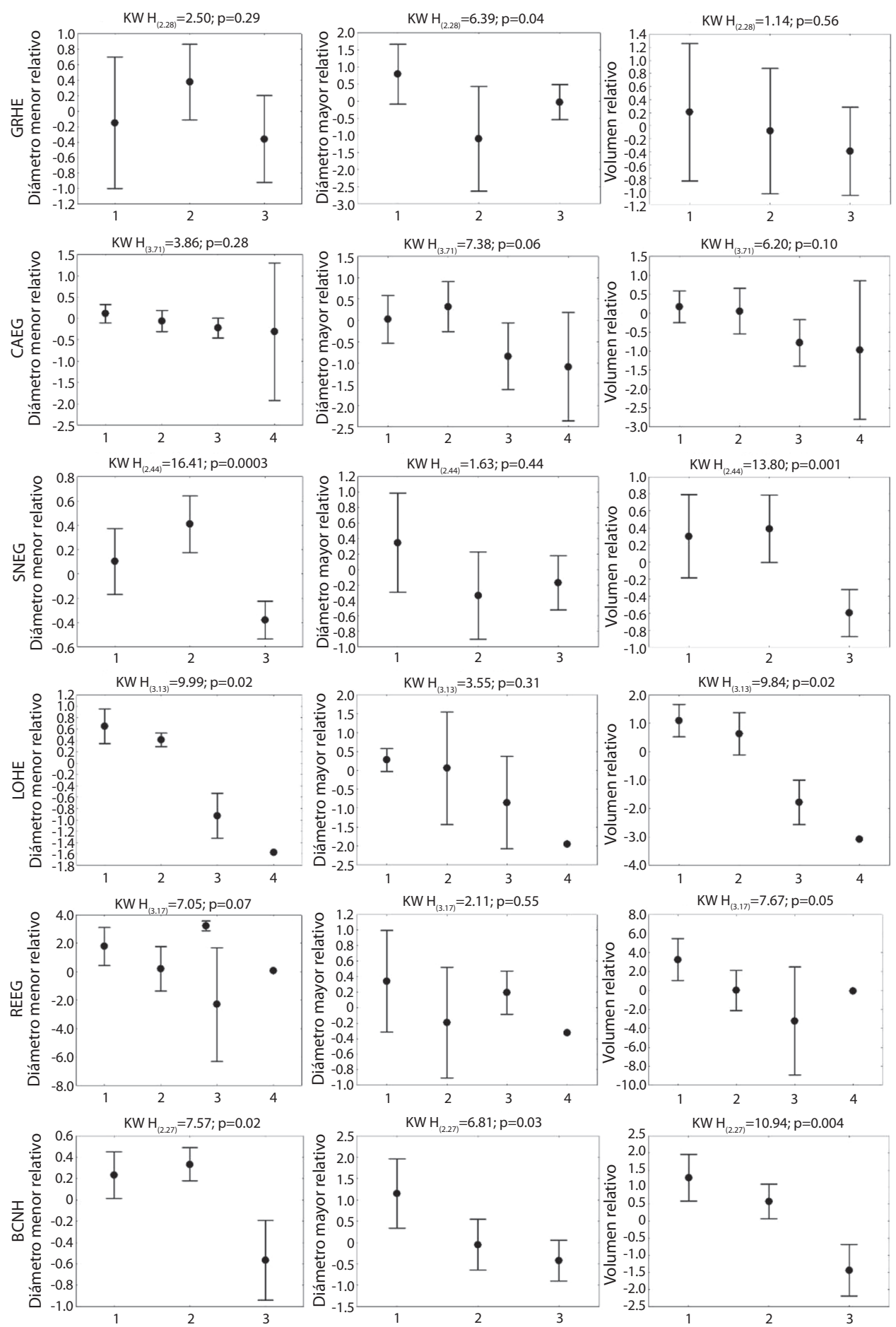

Fig. 5. Dimensiones de los huevos según el orden de puesta, relativizadas al promedio de la nidada, en cinco especies de garzas (Aves: Ardeidae) en la ciénaga de Birama, Cuba (tamaños de muestra y siglas de las especies en el cuadro 2).

Fig. 5. External dimensions of eggs by laying order, relativized to the mean of the clutch, in five species of egrets and herons (Aves: Ardeidae) in the Birama Swamp, Cuba (samples sizes and species symbols in table 2). 

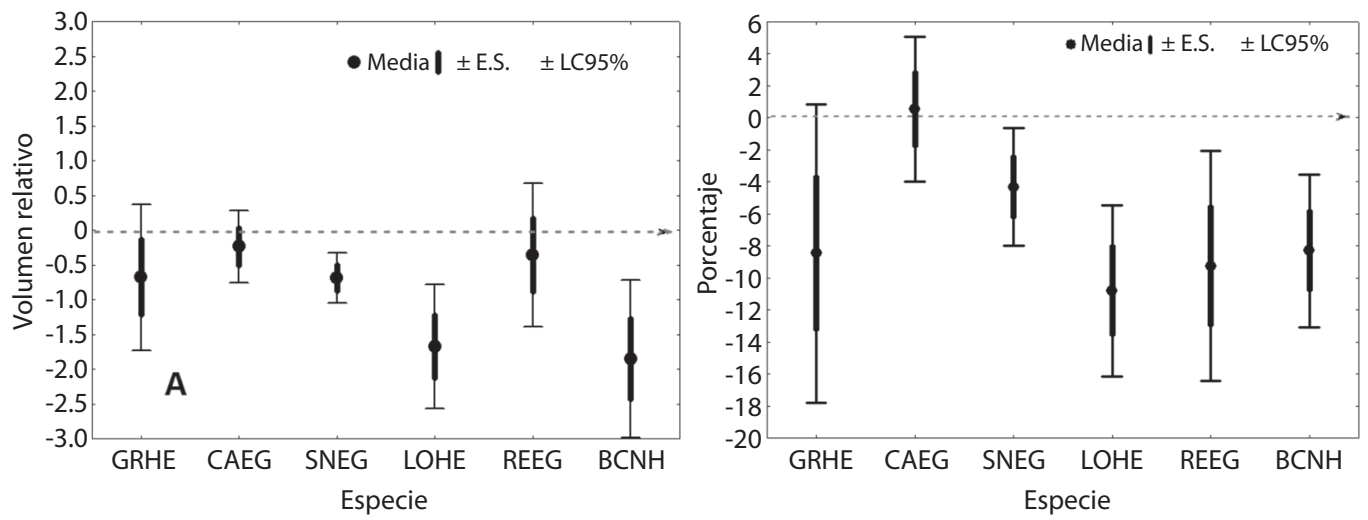

Fig. 6. Diferencias absolutas en el volumen del tercer huevo (A) con el promedio de la nidada y (B) con el primer huevo en seis especies de garzas (Aves: Ardeidae) en la ciénaga de Birama, Cuba (valores relativizados al peso corporal adulto, expresados en porcentaje) (las siglas de las especies aparecen en el cuadro 2).

Fig. 6. Absolute differences in egg volume in the third egg (A) against clutch average and (B) against the first egg, in six species of egrets and herons (Aves: Ardeidae) in the Birama Swamp, Cuba (values are relativized to adult body weight, and expressed as percentage) (species symbols in Table 2).
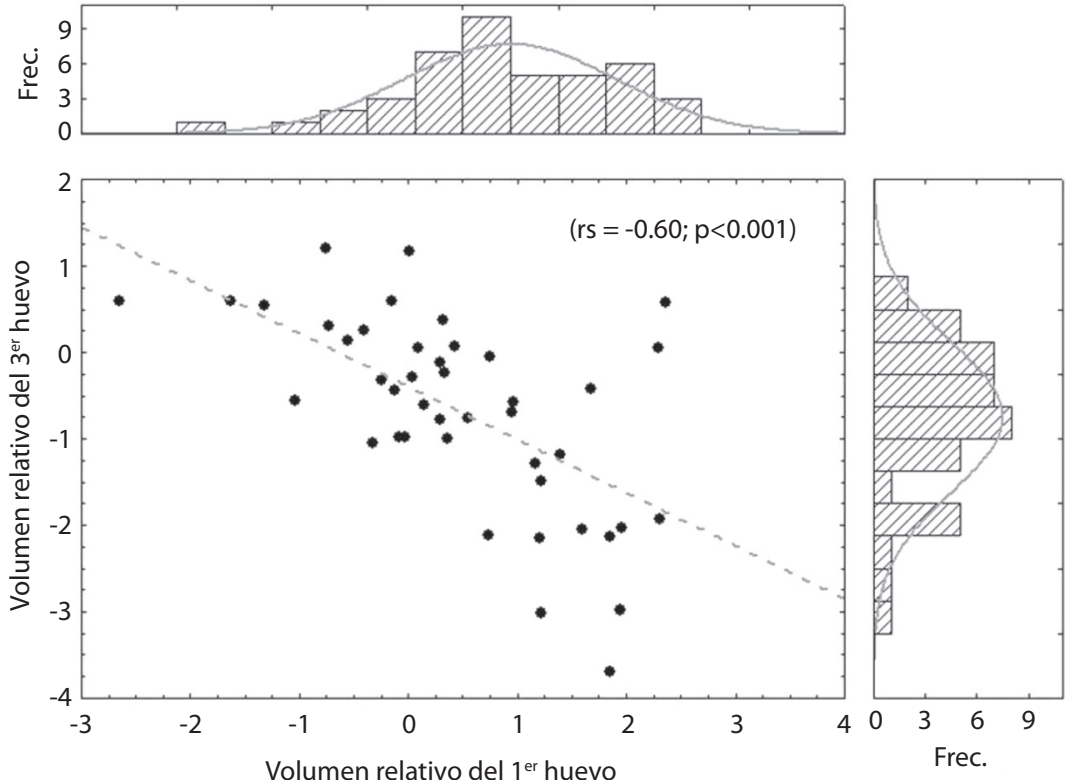

Fig. 7. Relación entre el volumen relativo del primer y el tercer huevo en las nidadas de ocho especies de garzas (Aves: Ardeidae) en la ciénaga de Birama, Cuba.

Fig. 7. Relationship between relative volume of the first and third egg in the clutch of eight species of herons and egrets (Aves: Ardeidae) in the Birama Swamp, Cuba. 


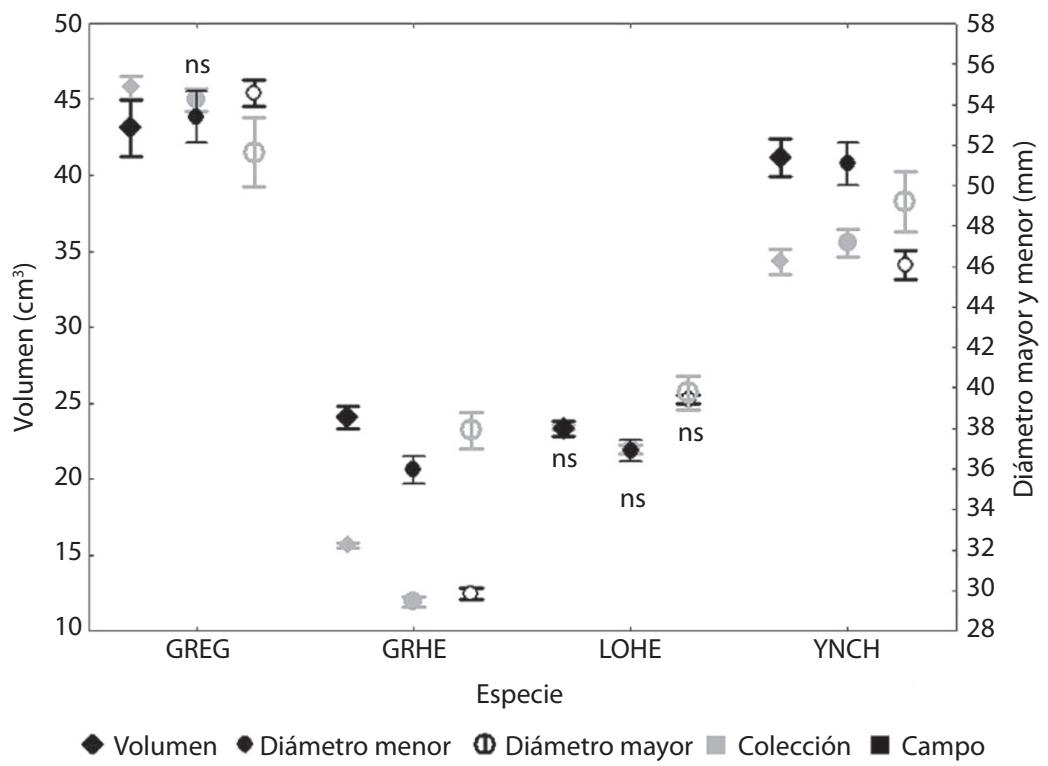

Fig. 8. Diferencias en las dimensiones de los huevos entre datos de campo y de colecciones en cuatro especies de garzas (Aves: Ardeidae) (ns: diferencias no significativas) (las siglas de las especies aparecen en el cuadro 2).

Fig. 8. Differences in egg sizes between field data and scientific collections in four species of herons and egrets (Aves: Ardeidae) (ns: non- significant differences) (species symbols in Table 2).

encontró dimensiones medias de $45.26 \pm 2.40 \mathrm{x}$ $32.98 \mathrm{~mm} \pm 1.09$ en 275 huevos de $B$. ibis seleccionados aleatoriamente en Texas.

Dentro de los patrones de variación en las dimensiones de los huevos de las aves, uno de los mejor establecidos es que, al comparar diferentes especies, su tamaño varía fundamentalmente en relación con la masa corporal del individuo adulto y su modo de desarrollo. Aunque esta tendencia ha sido estudiada principalmente analizando especies entre familias u órdenes diferentes, los resultados demuestran que se mantiene dentro de la misma familia Ardeidae. La proporción del huevo con el peso corporal se mantiene dentro de la norma descrita, donde la tendencia general es a que se mantengan entre un $2-11 \%$ de la masa corporal del adulto, salvo excepciones.

No existen diferencias interespecíficas marcadas en la variabilidad de las dimensiones de los huevos, que se mantuvo entre 6 y $9 \%$ en el volumen, ligeramente superior al reportado por Viñuela (1997) para Milvus migrans (4.4-5.0\%). En limícolas se ha reportado que el tamaño de los huevos tiene coeficientes de variación muy bajos, entre el 5-7\% (Miller, 1979; Ricklefs, 1984), influido porque estas especies son precociales, y no existe ningún patrón consistente de variación asociado al orden de puesta (Nol, \& Lambert, 1984; Slagsvold et al., 1984; Nol et al., 1997). En las garzas estudiadas la variación de las dimensiones de los huevos teniendo en cuenta la variabilidad intra-nidada y solo entre nidos fue semejante en la mayoría de los casos, excepto para el diámetro menor, en el cual a pesar de ser menor en magnitud, fue marcadamente diferente en ambos casos en $B$. virescens, E. rufescens y $E$. caerulea. La menor variabilidad del diámetro menor está relacionada a la constricción que sobre este impone la capacidad del oviducto y de la cloaca de las hembras (Viñuela, 1997).

Las diferencias en orden de puesta y entre nidos se ha estimado que explican entre el $69-79 \%$ de la variación total en el volumen de los huevos en N. nycticorax, E. thula y E. alba (Custer, \& Frederick, 1990). La existencia de un patrón de asimetría direccional consistente 
intra-nidada debe incrementar el coeficiente de variación cuando se utilizan las dimensiones individuales de los huevos por encima de cuando se toman los valores promedio de los nidos. Esto sin embargo, no es evidente en el caso del $B$. virescens, lo cual indica un mayor efecto de la hembra sobre la variabilidad de este carácter.

Se ha mencionado repetidamente que el tamaño de los huevos puede estar limitado por restricciones nutricionales de la hembra, y por tanto, relacionarse negativamente con el tamaño de puesta. Blackburn (1991) encontró que el tamaño de puesta es capaz de explicar hasta el $20 \%$ de la variación en tamaño del huevo en un grupo de 1530 especies de aves. En las especies estudiadas este hecho no fue evidente y la tendencia encontrada no parece relacionarse a diferencias etarias, ya que, al menos en $B$. ibis ha sido descrito que los nidificantes más inexpertos suelen ponen huevos menores (Telfair, 1983), al igual que en otras especies de aves (Viñuela, 1997). Syderman y Emslie (1992) mencionan que con la edad de los padres el volumen del huevo aumenta, las diferencias intra-nidada se hacen menos marcadas y las puestas se hacen más sincrónicas, sin afectarse el éxito de cría. De cualquier forma, el empleo del volumen del huevo no es suficiente como medida de su calidad (Arnold, \& Green, 2007).

En relación a la estrategia reduccionista de nidada, el mantenimiento del patrón de jerarquía de tallas entre hermanos, que permite la mortalidad diferencial, parece depender de varios factores sinérgicos, algunos de los cuales actúan directamente sobre el tamaño relativo del último huevo (Parsons, 1970; Nisbet, 1978). Este menor tamaño relativo puede depender del agotamiento de las reservas (Ryden, 1978; Pierotti, \& Bellrose, 1986; Sydeman, \& Emslier, 1992), aunque varias hipótesis alternativas han sido propuestas. En las garzas la variación, sin embargo, es muy pequeña en comparación con el tamaño adulto, por lo que energéticamente es despreciable y es poco probable que el patrón observado dependa de restricciones nutricionales. Este patrón, a pesar de ello, debe tener otro papel funcional en el establecimiento de la jerarquía intra-nidada, al ser más pronunciada la asimetría en especies con estrategia reduccionista de nidada (Slagsvold et al., 1984). Esto no implica que pueda descartarse totalmente alguna influencia nutricional, ya que la composición química más que su volumen- también puede cambiar con el orden de puesta (Ricklefs, 1977). De cualquier forma, la correlación entre el tamaño de los huevos y la disponibilidad de alimentos debe ser vista con cuidado, ya que un pulso en los recursos tróficos puede también acelerar la puesta y aumentar el número de huevos por nidada (Viñuela, 1997).

A partir de la consistencia en el patrón observado de disminución de la talla en la secuencia de puesta, Custer y Frederick (1990) señalaron la posibilidad de determinar el orden de puesta del huevo a partir del patrón de tallas en E. thula, E. alba y N. nycticorax. Sin embargo, sus resultados no fueron muy consistentes en todos los casos. Los huevos en nidadas de dos no fueron diferentes entre ellos en relación al orden de puesta, y en nidadas de tres huevos solo el último fue significativamente menor. Resultados similares fueron encontrados en la ciénaga de Birama en estas mismas especies. En E. thula solo el tercer huevo tuvo un volumen relativamente menor y la alta variabilidad de los diámetros mayores y menores impiden cualquier estimación precisa del orden de puesta. Un patrón similar apareció en $E$. tricolor, mientras que en $B$. ibis y $B$. virescens no fue detectable a través de la alta variabilidad de las medidas.

Los patrones de variación en la talla de los huevos en este grupo de especies distan de estar completamente esclarecidos. La variabilidad de estas medidas es demasiado alta para obtener resultados estadísticamente confiables, consistentes entre localidades o especies, por lo cual se requieren de muchos más estudios en diferentes condiciones. Posiblemente, solo un meta-análisis bien diseñado de los resultados descritos en un amplio conjunto de localidades, años y especies, pueda brindar la suficiente potencia y arrojar luz sobre este fenómeno. 


\section{AGRADECIMIENTOS}

Buena parte del trabajo de campo fue posible gracias al apoyo de la Empresa Nacional para la Protección de la Flora y la Fauna, y en especial a los trabajadores del Refugio de Fauna Delta del Cauto. Se agradece a Idea Wild por proveer equipamiento e instrumentos de medición y a un gran número de estudiantes de la carrera de Biología que participaron en la toma de datos.

\section{RESUMEN}

Las variaciones intranidada de la talla de los huevos de las aves responden a numerosos factores ecológicos y de la historia evolutiva de las especies. En las zancudas aunque se ha descrito una disminución de la talla con el orden de puesta, los trabajos comparativos entre especies son escasos. En este trabajo se describen los patrones de variación del tamaño de los huevos de nueve especies de garzas (Aves: Ardeidae) nidificantes en la ciénaga de Birama, Cuba. Los patrones se describen a partir de las dimensiones de 3142 huevos, pertenecientes a 1875 nidos de Butorides virescens, Bubulcus ibis, Ardea alba, Nycticorax nycticorax, Nyctanassa violacea y cuatro especies de Egretta, tomados en la ciénaga de Birama, Cuba, entre 1998 y 2006. Los huevos representaron entre el 4.9-10\% del peso adulto y tuvieron un coeficiente de variación del volumen entre el 6-9\%. No existió una relación general consistente entre especies entre el tamaño de puesta y las dimensiones de los huevos. El volumen promedio del huevo tiende a disminuir con el orden de puesta, pero no es estadísticamente detectable en Butorides y Bubulcus. El último huevo fue entre un 0.2 y un $15 \%$ menor que el primero, disminuyendo su volumen relativo con la talla del primer huevo. La asimetría intranidada es menos marcada en E. thula y prácticamente fluctuante alrededor de cero en Bubulcus. En general, la talla del huevo solo puede predecir el orden de puesta para el último huevo en nidadas mayores de dos con un $72.4 \%$ de confianza.

Palabras clave: tamaño del huevo, variación intra-nidada, reproducción, zancudas coloniales, ardéidos, reducción de nidada.

\section{REFERENCIAS}

Arnold, T. W. (1999). What limits clutch size in waders? Journal of Avian Biology, 30, 216-220.

Arnold, T. W., \& Green, A. J. (2007). On the allometric relationship between size and composition of avian eggs: a reassessment. Condor, 109, 705-714.
Baichich, P. J., \& Harrison, C. J. O. (1997). A guide to the nests, eggs, and nestlings of North American birds. Nueva York: Acad. Press.

Bent, A. C. (1926). Life histories of North American marsh birds. U.S. Natl. Mus. Bull. No. 135.

Blackburn, T. M. (1991). An interspecific relationship between egg size and clutch size in birds. Auk, 108, 973-977.

Custer, T. W., \& Frederick, P. C. (1990). Egg size and laying order of Snowy Egrets, Great Egrets, and Blackcrowned Night-Herons. Condor, 92, 772-775.

Denis, D. (2001). Dinámica metapoblacional en las colonias de garzas (Aves: Ardeidae) de la ciénaga de Biramas, Cuba. Journal of Caribbean Ornithology, 16(1), 35-44.

Denis, D., \& Rodríguez, A. (2007). Variación en la composición interna de los huevos de seis especies de zancudas en la ciénaga de Birama, Cuba. Journal of Caribbean Ornithology, 20, 26-34.

Denis, D., \& Ponce de León, J. L. (2006). Ecología reproductiva de la Garza Rojiza Egretta rufescens (Aves: Ardeidae) en la ciénaga de Birama. Biología, 20(1-2), 17-23.

Denis, D., \& Ponce de León, J. L. (2007). Variación del grosor de la cáscara del huevo en diez especies de zancudas (Aves: Ciconiformes) en la ciénaga de Birama, Cuba. Journal of Caribbean Ornithology, 20, 17-25.

Denis, D., Rodríguez, A., Rodríguez, P., \& Jiménez, A. (2003). Reproducción de la Garza Ganadera (Bubulcus ibis) en la Ciénaga de Biramas, Cuba. Journal of Caribbean Ornithology, 16(1), 45-54.

Denis, D.; Torrella, L., Rodríguez, P., \& Rodríguez, A. (1999). Nuevo reporte de nidificación de la Garza Rojiza (Egretta rufescens) en Cuba. El Pitirre, 12(3), 96-97.

Denis, D., Olavarrieta, U., \& Andraca, L. (2009). Actualización de la constante de Hoyt para el cálculo del volumen de los huevos en garzas cubanas (Aves: Ciconiiformes). Biología, 22(1-2), 75-77.

Dickerman, R. W., \& Gavino, G. T. (1969). Studies of a nesting colony of Green Herons at San Blas, Nayarit, Mexico. Living Bird, 8, 95-111.

Dusi, J. L. (1966). The identification characters of nests, eggs and nestlings of some herons, ibises and Anhingas. Alabama Birdlife, 14, 2-8.

Frederick, P. C., \& Collopy, M. W. (1988). Reproductive ecology of wading birds in relation to water conditions in the florida everglades. FL. Cooperative Fish and Wildlife Research Unit, Univ. of Florida, Gainesville. 
Hammatt, R. B. (1981). Reproductive biology in a Louisiana estuarine heronry (Master's thesis). Louisiana State Univ., Baton Rouge.

Hoyt, D. F. (1979). Practical methods of estimating volume and fresh weight of bird eggs. Auk, 96, 73-77.

Huey, L. M. (1926). A prolific Anthony Green Heron. Condor, 28, 94-96.

Jover, L.; Ruiz, X., \& González-Martín, M. (1993). Significance of intraclutch egg size variation in the Purple Heron. Ornis Scandinavica, 24(2), 127-134.

Lack, D. (1968). Ecological adaptations for breeding in birds. Londres, U.K.: Methuen.

Meyerriecks, A. J. (1962). Green Heron. In R. S. Palmer (Ed.). Handbook of North American birds (Vol. 1, pp. 419-426). New Haven, CT.: Yale Univ. Press.

Miller, E. H. (1979). Egg size in the Least Sandpiper Calidris minutilla on Sable Island, Nova Scotia, Canada. Ornis Scandinavica, 10, 10-16.

Mock, D. W., \& Parker, A. (1986). Advantages and disadvantages of egrets and heron brood reduction. Evolution, 40, 459-470.

Nisbet, I. C. T. (1978). Dependence of fledging success on egg-size, parental performance and egg-composition among Common and Roseate terns, Sterna hirundo and S. dougallii. Ibis, 120, 207-215.

Nol, E., \& Lambert, A. (1984). Comparison of Killdeers, Charadrius vociferus, breeding in mainland and peninsular sites in southern Ontario. Canadian Field Naturalist, 98, 7-11.

Nol, E., Blanken, M. S., \& Flynn, L. (1997). Sources of variation in clutch size, egg size and clutch completion dates of semipalmated plovers in Churchill, Manitoba. Condor, 99, 389-396.
Palmer, R. G. (1962). Handbook of North American birds. Vol. 1: loons through flamingos. New Haven, CT.: Yale Univ. Press.

Parson, J. (1970). Relationship between egg size and posthatching chick mortality in the Herring Gull (Larus argentatus). Ibis, 117, 517-520.

Pierotti, R., \& Bellrose, C. A. (1986). Proximate and ultimate causation of egg size and the "third-chick disadvantage" in the western gull. Auk, 103, 401-407.

Ricklefs, R. E. (1977). Composition of eggs of several birds' species. Auk, 94, 350-356.

Ricklefs, R. E. (1984). Egg dimensions and neonatal mass of shorebirds. Condor, 86, 7-11.

Ryden, O. (1978). Egg weight in relation to laying secuence in a south Swedish urban population of the Blackbird Turdus merula. Ornis Scandinavica, 9, 172-177.

Slagsvold, T., Sandvik, J., Rofstad, G., Lorensten, O., \& Husby, M. (1984). On the adaptative value of intraclutch egg size variation in birds. Auk, 101, 685-697.

StatSoft, Inc. (2007). STATISTICA (data analysis software system). Versión 8.0. www.statsoft.com.

Stoleson, S. H., \& Beissinger, S. R. (1995). Hatching asynchrony and the onset of incubation in birds, revisited: when is the critical period? Current Ornithology, 12, 191-270

Sydeman, W. J., \& Emslie, S. D. (1992). Effects of parental age on hatching asynchrony, egg size and the third chick disadvantage in Western Gulls. Auk, 109, 242-248.

Telfair, R. C., II. (1983). The cattle egret: a Texas focus and world view. Dept. of Wildl. and Fish. Sci., Texas A\&M Univ.

Viñuela, J. (1997). Adaptation vs. constraint: intraclutch egg-mass variation in birds. Journal of Animal Ecology, 66, 781-792. 